\title{
Some Results on SLAM and the Closing the Loop Problem
}

\author{
Agostino Martinelli, Nicola Tomatis and Roland Siegwart \\ Swiss Federal Institute of Technology Lausanne (EPFL) \\ CH-1015 Lausanne, Switzerland \\ e-mail: agostino.martinelli, nicola.tomatis, roland.siegwart depfl.ch
}

\begin{abstract}
This paper addresses the closing loop problem as the challenge of using all the information from the observation gathered when closing the loop in order to optimally adjust the whole map (assuming a correct data association). The proposed approach is an approximation, which allows the calculation of the gain without keeping track of all the correlations (i.e. with a complexity independent of the number of the map elements). Furthermore, the paper presents an explicit mathematical demonstration showing that the correlations computed by the $E K F$-based SLAM are overestimated. More precisely, it is shown that these correlations decrease exponentially with respect to the heading error of the robot.

The approach is then empirically demonstrated by means of meaningful simulations. The results are then discussed and conclusions are pointed out in the last section.
\end{abstract}

Key Words: SLAM, Closing Loop Problem, Kalman filter, Sensor Fusion, Relative Observation

\section{INTRODUCTION}

Simultaneous Localization and Mapping (SLAM) requires a mobile robot to autonomously explore the environment with its on-board sensors, gain knowledge about it, interpret the scene, build an appropriate map and localize itself relative to this map.

A very successful method is the stochastic map approach. After the first precise mathematical definition of the stochastic map [11] early experiments ([5], [7]), have shown the quality of fully metric simultaneous localization and map building. One of the most discussed inconvenient of this approach is the computational requirement, which scales very badly (squarely) with the number of the map elements due to their correlations. To overcome this problem, several solutions have been proposed (e.g. [9], [12]).

Obviously, neglecting the correlations among the landmarks very far, could be a good approximation in the absence of large loops. Indeed, when the robot closes the loop, the correlations contain information about the whole loop which are needed in order to propagate the correction through the loop.

In this paper we introduce a method computing the necessary correlations between the landmarks and the robot in order to properly use all the information when closing the loop. We show that the linearization in the standard approach causes an overestimation of this information when the loop is large compared with the parameters characterizing the robot sensors. In section II we first compute the corrections on the map at the end of a loop as obtained from the standard approach. Starting from this computation, we suggest an approximation able to provide almost the same corrections but with a computational complexity independent of the number of map elements $(O(1)$ instead of $O\left(N^{2}\right)$ ). In section III we compute the same corrections by avoiding the linearization which characterizes the Extended Kalman Filter $(E K F)$ and therefore the $E K F$ based SLAM. The results obtained through simulations are presented in section IV. They compare the EKF-based SLAM to the approximation with $O(1)$ complexity. We believe that a complete real experiment would be required to evaluate the overall performance of these approaches. However, in this case, the simulation permits to more precisely compare the results when closing the loop. Finally, conclusions are presented in section $\mathrm{V}$.

\section{The Map Correction at the Closing Loop}

The innovation (defined as the difference between the observation and its prediction) related to the observation when the robot closes a loop, contains the information accumulated by the robot along the whole loop. This innovation can then be used to correct the configurations of all the landmarks in the loop. In this section we carry out the computation of the previous corrections. The section consists of two parts. In the former, we carry out the computation by adopting the standard solution to SLAM based on the $E K F$ [6], from now on $A M F$ (Absolute Map Filter). The $A M F$ requires a computational complexity which increases squarely with the number of landmarks in the loop. For this reason, in section II-B we suggest a possible solution able to correct the configuration of all the landmarks in the loop, whose complexity does not depend on the number of landmarks. It is possible to implement this solution when the exteroceptive sensor provides a relative observation between the robot and one landmark at a time.

For the sake of simplicity, we will refer in the following to the case of point landmark.

\section{A. Standard Solution}

In the $A M F$ the robot configuration and the location of each landmark are registered in one common global reference frame. An $E K F$ is used to estimate the state containing the previous global coordinates and its covariance matrix.

$$
X=\left[\begin{array}{llll}
X_{v}^{T} & X_{1}^{T} & \ldots & X_{N}^{T}
\end{array}\right]^{T}
$$




$$
P=\left[\begin{array}{cccc}
P_{v v} & P_{v 1} & \ldots & P_{v N} \\
P_{v 1}^{T} & P_{11} & \ldots & P_{1 N} \\
\ldots & \ldots & \ldots & \ldots \\
P_{v N}^{T} & P_{1 N}^{T} & \ldots & P_{N N}
\end{array}\right]
$$

where $X_{v}=[x, y, \theta]^{T}$ is the robot configuration, $X_{i}$ is the absolute location of the $i^{\text {th }}$ landmark, $P_{i j}$ is the crosscovariance between the $i^{t h}$ and $j^{t h}$ landmark location and $P_{v i}$ is the cross-covariance between the $i^{t h}$ landmark and the vehicle configuration. The "state transition equation" for the state $X$ restricted to the map part $\left(X_{i}\right)$ is the identity. Regarding the vehicle part, this equation is determined by the drive system of the robot. The $E K F$ is used to fuse the information coming from this transition equation with the information coming from an observational equation. This equation models the observation coming from an exteroceptive sensor and provides a vector depending on the state given in the equation (1).

$$
Z=h(X, w)
$$

where $w$ is a vector of temporally uncorrelated observation errors with zero mean and covariance matrix $R$.

When a relative observation between the robot and the landmark $i^{t h}$ occurs, the function in (3) will be

$$
Z=h(X, w) \equiv h\left(X_{v}, X_{i}, w_{i}\right)
$$

whose Jacobian with respect to the state in (1) will be

$$
H_{i}=\left[H_{X_{v}}, 0, \ldots, 0, H_{X_{i}}, 0, \ldots, 0\right]
$$

When the $E K F$ is adopted to update the state in (1) and its covariance in (2) by using this relative observation, the result is [6]:

$$
\begin{gathered}
X(k \mid k)=X(k \mid k-1)+W_{i}(k) \nu_{i}(k) \\
P(k \mid k)= \\
=P(k \mid k-1)-W_{i}(k) S_{i}(k) W_{i}^{T}(k)
\end{gathered}
$$

where

$$
\begin{gathered}
W_{i}(k)=P(k \mid k-1) H_{i}^{T}(k-1) S_{i}^{-1}(k) \\
S_{i}(k)=H_{i}(k-1) P(k \mid k-1) H_{i}^{T}(k-1)+R_{i}(k)
\end{gathered}
$$

and $X(k \mid k-1)$ and $P(k \mid k-1)$ are respectively the predicted state and its covariance (see equations (10) and (12) in [6]) and $\nu_{i}(k)$ is the innovation (equation (13) always in $[6])$.

By a direct computation it is easy to obtain from equations (5), (8) and (9)

$$
\begin{gathered}
P H_{i}^{T}=\left[\begin{array}{c}
P_{v v} H_{X_{v}}^{T}+P_{v i} H_{X_{i}}^{T} \\
P_{1 v} H_{X_{v}}^{T}+P_{1 i} H_{X_{i}}^{T} \\
\cdots \\
P_{i v} H_{X_{v}}^{T}+P_{i i} H_{X_{i}}^{T} \\
\cdots \\
P_{N v} H_{X_{v}}^{T}+P_{N i} H_{X_{i}}^{T}
\end{array}\right] \\
S_{i}=H_{X_{v}} P_{v v} H_{X_{v}}^{T}+H_{X_{v}} P_{v i} H_{X_{i}}^{T}+ \\
+H_{X_{i}} P_{i v} H_{X_{v}}^{T}+H_{X_{i}} P_{i i} H_{X_{i}}^{T}+R_{i}
\end{gathered}
$$

where, for the sake of simplicity, we omitted the time $k$ and in particular we indicated with $P$ the predicted covariance $P(k \mid k-1)$.

The previous equations allow to compute the Kalman gain on all the landmarks in the map and on the robot when a relative observation between the $i^{\text {th }}$ landmark and the robot occurs.

Let us consider now the closing loop problem and let us suppose that the $m^{\text {th }}$ landmark was the first one observed in the loop. This means that all the landmarks in the loop were introduced into the map after the $m^{t h}$ landmark. We assume that the error on the $m^{t h}$ landmark is zero (i.e the matrix $P_{m m}$ is the null matrix). This corresponds to put a new reference frame on this first landmark. Our assumption implies that also each block element $P_{m j}$ is null.

When the loop is closed, namely the $m^{\text {th }}$ landmark is observed again, the Kalman gain can be computed for each map element starting from the equations (10) and (11). We obtain:

$$
\begin{gathered}
P H_{m}^{T}=\left[\begin{array}{c}
P_{v v} H_{X}^{T} \\
P_{1 v} H_{X_{v}}^{T} \\
\ldots \\
P_{j v} H_{X_{v}}^{T} \\
\cdots \\
0 \\
\cdots \\
P_{N v} H_{X_{v}}^{T}
\end{array}\right] \\
S_{m}=H_{X_{v}} P_{v v} H_{X_{v}}^{T}+R_{m}
\end{gathered}
$$

where the line with 0 in (12) corresponds, clearly, to the Kalman gain on the $m^{\text {th }}$ landmark perfectly known at the beginning.

From the two previous equations it is possible to conclude that, in order to use the information coming from the observation at the closing loop, it is sufficient to maintain at each time step the blocks in the covariance matrix containing the robot $\left(P_{v v}\right.$ and $P_{v j}$ ). In the $A M F$ it is not possible to separate these blocks from the rest of the covariance matrix in (2). Indeed, from (7) it is possible to see that the update for $P_{v v}$ and $P_{v j}$ requires the update of the other blocks. In II-B we suggest an approximated method to update only $P_{v v}$ and $P_{v j}$. 


\section{B. Approximated Solution}

The motion of the robot can be described through the unicycle model, i.e. through the following differential equations:

$$
\begin{gathered}
\dot{x}_{v}=v \cos \theta_{v} \\
\dot{y}_{v}=v \sin \theta_{v} \\
\dot{\theta}_{v}=\omega
\end{gathered}
$$

where the knowledge of the robot configuration at a given time and of the function $v(\tau)$ and $\omega(\tau)$ are required to know the robot configuration at a subsequent time. Let us suppose that at the time $t_{*}$ the robot configuration is $\left[x_{*}, y_{*}, \theta_{*}\right]^{T}$. By integrating the equations (14-16) we obtain for the time $t(t>$ $\left.t_{*}\right)$ :

$$
\begin{gathered}
x_{v}(t)=x_{*}+A\left(t, t_{*}\right) \cos \theta_{*}-B\left(t, t_{*}\right) \sin \theta_{*} \\
y_{v}(t)=y_{*}+A\left(t, t_{*}\right) \sin \theta_{*}+B\left(t, t_{*}\right) \cos \theta_{*} \\
\theta_{v}(t)=\theta_{*}+K\left(t, t_{*}\right)
\end{gathered}
$$

where

$$
\begin{gathered}
K\left(t, t_{*}\right)=\Omega(t)-\Omega\left(t_{*}\right) \\
A\left(t, t_{*}\right)=\cos \Omega\left(t_{*}\right)\left[C(t)-C\left(t_{*}\right)\right]+ \\
+\sin \Omega\left(t_{*}\right)\left[S(t)-S\left(t_{*}\right)\right] \\
B\left(t, t_{*}\right)=\cos \Omega\left(t_{*}\right)\left[S(t)-S\left(t_{*}\right)\right]- \\
-\sin \Omega\left(t_{*}\right)\left[C(t)-C\left(t_{*}\right)\right]
\end{gathered}
$$

and

$$
\begin{gathered}
\Omega(t)=\int_{t_{0}}^{t} \omega(\tau) d \tau \\
S(t)=\int_{t_{0}}^{t} v(\tau) \sin \Omega(\tau) d \tau \\
C(t)=\int_{t_{0}}^{t} v(\tau) \cos \Omega(\tau) d \tau
\end{gathered}
$$

where $t_{0}$ can be any time before $t_{*}$.

From the equations (20-25) it is possible to express $A\left(t, t_{*}\right)$ and $B\left(t, t_{*}\right)$ as functions of the robot translations $(v(\tau) d \tau)$ and rotations $(\omega(\tau) d \tau)$ occurred after $t_{*}$. We have:

$$
A\left(t, t_{*}\right)=\int_{t_{*}}^{t} v(\tau) \cos \left(\int_{t_{*}}^{\tau} \omega\left(\tau^{\prime}\right) d \tau^{\prime}\right) d \tau=
$$

$$
\begin{gathered}
=\int_{t_{*}}^{t} v(\tau) \cos K\left(\tau, t_{*}\right) d \tau \\
B\left(t, t_{*}\right)=\int_{t_{*}}^{t} v(\tau) \sin \left(\int_{t_{*}}^{\tau} \omega\left(\tau^{\prime}\right) d \tau^{\prime}\right) d \tau= \\
=\int_{t_{*}}^{t} v(\tau) \sin K\left(\tau, t_{*}\right) d \tau
\end{gathered}
$$

The $A M F$ estimates at each time step the robot configuration. From two subsequent robot configurations it is possible to obtain the robot translation and the robot rotation occurred during the considered step. Since these displacements are shift and rotation invariant, in some cases it can be reasonable to assume that they are independent of a landmark previously observed by the robot. This assumption is obviously an approximation. However, when this landmark is very far from the robot this assumption is satisfied. Therefore, a distance $D$ is introduced: when the distance between the robot and the landmark is larger than $D$ the approximation is valid. Clearly, the parameter $D$ depends on the parameters characterizing the robot sensors, both exteroceptive and proprioceptive.

If we use the previous approximation, in order to maintain the block element $P_{v v}$, it is not necessary to implement the fully correlated $A M F$ (i.e. update all the $N^{2}$ correlations among the landmarks). Indeed, the same result can be achieved if, at each step, only the landmarks whose distance from the robot is smaller than $D$ are included in the $E K F$. We call this $E K F$ the Local SLAM ( $L S L A M)$. Due to its locality, the complexity of $L S L A M$ does not depend on the number of landmarks.

In section II-A we showed that to use the information coming from the observation at the closing loop, it is necessary to know both $P_{v v}$ and $P_{v j}$. In the following, we provide the expression for the latter (since $L S L A M$ provide automatically the former).

Let us indicate with $t_{j}$ the time when the distance between the robot and the $j^{\text {th }}$ landmark is equal to $D$. This means that, starting from $t_{j}$, the robot translation and the robot rotation estimated through $L S L A M$ are independent of the $j^{t h}$ landmark. The robot configuration at the time $t$ is given by the equations (17-19) where $t_{*}=t_{j}$. Since the quantities $A\left(t, t_{j}\right), B\left(t, t_{j}\right)$ and $K\left(t, t_{j}\right)$ contain only the displacements estimated through $L S L A M$ after $t_{j}$ (see the equations (26), (27) and (20)), they are independent of the $j^{\text {th }}$ landmark. Therefore, $P_{v j}$ can be easily obtained by linearizing (17-19) in $\left[x_{*}, y_{*}, \theta_{*}\right]^{T}=\left[x\left(t_{j}\right), y\left(t_{j}\right), \theta\left(t_{j}\right)\right]^{T}$. We obtain:

$$
P_{v j}=F\left(t, t_{j}\right) P_{v j}\left(t_{j}\right)
$$

where $P_{v j}\left(t_{j}\right)$ is the covariance at time $t_{j}$ estimated by $L S L A M$ and the matrix $F\left(t, t_{j}\right)$ is the Jacobian of the function in (17-19) in $\left[x\left(t_{j}\right), y\left(t_{j}\right), \theta\left(t_{j}\right)\right]^{T}$

$$
F\left(t, t_{j}\right)=
$$




$$
=\left[\begin{array}{ccc}
1 & 0 & -A\left(t, t_{j}\right) \sin \theta\left(t_{j}\right)-B\left(t, t_{j}\right) \cos \theta\left(t_{j}\right) \\
0 & 1 & A\left(t, t_{j}\right) \cos \theta\left(t_{j}\right)-B\left(t, t_{j}\right) \sin \theta\left(t_{j}\right) \\
0 & 0 & 1
\end{array}\right]
$$

By using these last equations it is possible to compute $P_{v j}$. The computation requires to update the function in (23-25). Therefore, the complexity is independent of the number of landmarks.

\section{COMPutation OF THE CORRElations Without LINEARIZATION}

The computation carried out in the previous section is based on two approximations: the former is the introduction of the $L S L A M$ instead of the $A M F$. The latter is the linearization. This second approximation is present also in the $A M F$. Indeed, the $A M F$ is based on the $E K F$ which requires to linearize the equations characterizing the dynamics and the observation of the system [2]. The problems arising from this linearization are not negligible for $S L A M$ (see for example [3]).

In the following we compute the block $P_{v j}$ by avoiding the linearization. We will show that the result depends dramatically on the error on the robot orientation. On the other hand, the error in the orientation is the element $(3,3)$ in the block $P_{v v}$. This last covariance is estimated through LSLAM.

We have

$$
P_{v j} \equiv\left\langle[\tilde{x}(t), \tilde{y}(t), \tilde{\theta}(t)]^{T} \tilde{X}_{j}\right\rangle
$$

where we indicate with the error on the corresponding quantity (that is the difference between the true (unknown) value and its estimated mean value).

To proceed we have to use the equations (17-19) with $t_{*}=$ $t_{j}$ and $\left[x_{*}, y_{*}, \theta_{*}\right]^{T}=\left[x\left(t_{j}\right), y\left(t_{j}\right), \theta\left(t_{j}\right)\right]^{T}$. We obtain for the three lines of $P_{v j}$ respectively:

$$
\begin{aligned}
&\left\langle\tilde{x}(t) \tilde{X}_{j}\right\rangle=\left\langle\tilde{x}\left(t_{j}\right) \tilde{X}_{j}\right\rangle- \\
&-A\left(t, t_{j}\right) \sin \theta\left(t_{j}\right) \aleph-B\left(t, t_{j}\right) \cos \theta\left(t_{j}\right) \aleph \\
&\left\langle\tilde{y}(t) \tilde{X}_{j}\right\rangle=\left\langle\tilde{y}\left(t_{j}\right) \tilde{X}_{j}\right\rangle+ \\
&+A\left(t, t_{j}\right) \cos \theta\left(t_{j}\right) \aleph-B\left(t, t_{j}\right) \sin \theta\left(t_{j}\right) \aleph \\
&\left\langle\tilde{\theta}(t) \tilde{X}_{j}\right\rangle=\left\langle\tilde{\theta}\left(t_{j}\right) \tilde{X}_{j}\right\rangle
\end{aligned}
$$

where the quantities $\left\langle\tilde{x}\left(t_{j}\right) \tilde{X}_{j}\right\rangle,\left\langle\tilde{y}\left(t_{j}\right) \tilde{X}_{j}\right\rangle$ and $\left\langle\tilde{\theta}\left(t_{j}\right) \tilde{X}_{j}\right\rangle$ are the three lines of the matrix $P_{v j}\left(t_{j}\right)$ (estimated by $L S L A M$ ) and $\aleph$ indicate the following mean value:

$$
\aleph=\left\langle\sin \tilde{\theta}\left(t_{j}\right) \tilde{X}_{j}\right\rangle
$$

The previous result is obtained by observing that $\left\langle\cos \tilde{\theta}\left(t_{j}\right) \tilde{X}_{j}\right\rangle=[0,0]$ since the function $\cos$ is an even function. Regarding the integral appearing in (34) we obtain through a direct computation [10]:

$$
\aleph=\left\langle\tilde{\theta}\left(t_{j}\right) \tilde{X}_{j}\right\rangle \exp \left[-\frac{\sigma_{\theta}^{2}\left(t_{j}\right)}{2}\right]
$$

where $\sigma_{\theta}^{2}\left(t_{j}\right)$ is the error in the robot orientation at the time $t_{j}$.

By comparing equations (28-29) with the equations (30-35) we note that the linearization approximates the exponential term in (35) with 1 . This results in an overestimation on the blocks $P_{v j}$ and consequently, the corrections at the closing loop on all the map elements, will be erroneous because actually a not negligible amount of the information is lost. When the exponential term is included, the corrections will be smaller.

\section{Results AND Discussion}

In this section we compare the results obtained by using the $A M F$ with the approximated solution introduced in IIB. Since the aim is to point out the differences among the previous approaches for the estimation at the moment when the loop is closed, the simulations are more appropriate than a complete experiment. However, in order to validate the overall performance a complete experiment would be necessary.

\section{A. Simulated Environment}

In our simulated experiment the data coming from the encoder sensors were delivered at the frequency of $1 \mathrm{KHz}$, in agreement with the experiments carried out on real platforms in our laboratory (e.g. [1]). The simulated robots are equipped with a differential drive system. We adopted the same odometry error model introduced in [4] where the actual translation of the right and left wheel at a given time step is assumed to be a gaussian random variable satisfying the following relation:

$$
\begin{gathered}
\delta \rho^{R / L}=\overline{\delta \rho}^{R / L}+\nu^{R / L} \\
\overline{\delta \rho}^{R / L}=\delta \rho^{e R / L} \delta_{R / L} \\
\nu^{R / L} \sim N\left(0, K_{R / L}\left|\delta \rho^{e R / L}\right|\right)
\end{gathered}
$$

In other words, both $\delta \rho^{R}$ and $\delta \rho^{L}$ are assumed to be gaussian random variables, whose mean values are given by the encoder readings (respectively $\delta \rho^{e R}$ and $\delta \rho^{e L}$ ) corrected for the systematic errors (which are assumed to increase linearly with the distance travelled by each wheel), and whose variances also increase linearly with the travelled distance. Furthermore, it is assumed that $\delta \rho^{R}$ and $\delta \rho^{L}$ are uncorrelated. In our simulation we adopted $\delta_{R}=\delta_{L}=1$ (i.e. encoders perfectly calibrated) and $K_{R}=K_{L}=510^{-5} \mathrm{~m}$ (that is the value experimentally estimated for our robot in our laboratory, [8]).

The exteroceptive sensor provides the bearing angles and the distances of the 6 landmarks closest to the robot. The frequency for this simulated sensor is $1 \mathrm{~Hz}$. The errors on 


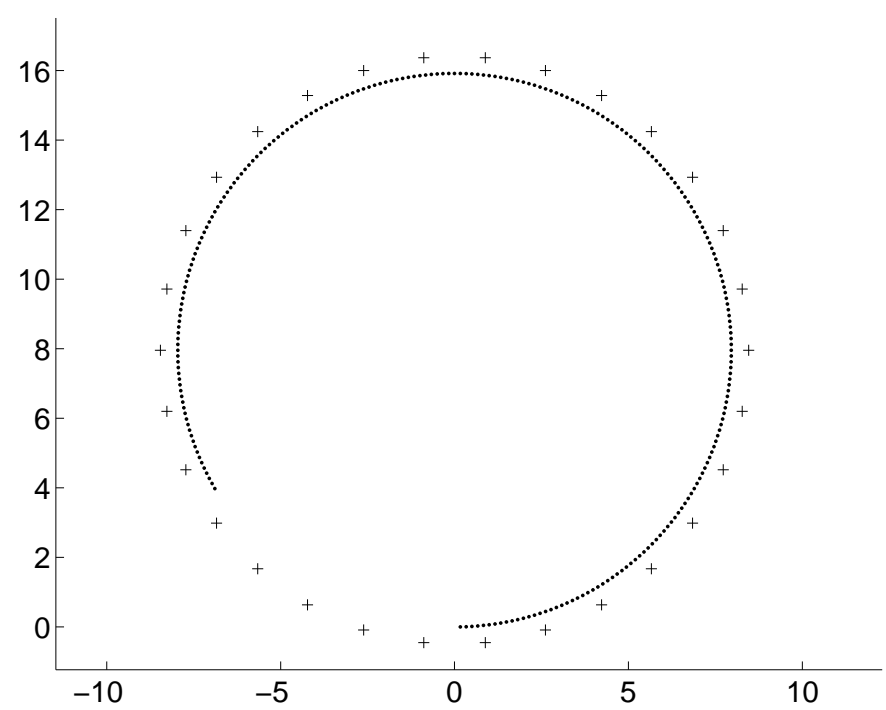

Fig. 1. The simulated experiment. The lines represent the robot trajectories; + represent the landmark positions. The unities are $m$.

the bearing and the distance are independent and respectively with the variances: $\sigma_{b}^{2}=\left(1^{o}\right)^{2}$ and $\sigma_{d}^{2}=(0.02 m)^{2}$. The robot speed is $0.2 \mathrm{~m} / \mathrm{s}$.

In the simulated experiment, the robot moves along a circumference. The number of landmarks is 30 . The motion of the robot is interrupted when the first landmark is observed again after the loop. The trajectory and the landmarks are displayed in figures 1 . The length of the path followed by the robot is $50 \mathrm{~m}$.

Instead of introducing the distance $D$ introduced in II-B to define the locality of $L S L A M$, we characterize this locality by fixing the maximum number of elements for the state estimated through $L S L A M$. In particular, this state contains the closest 10 landmarks to the robot.

\section{B. Results}

In the figures 2 and 3 we plot the mean error on the estimated landmark positions vs the distance travelled by the robot. We do not observe any relevant difference among the two approaches before and after closing the loop. In particular, the final error is slightly smaller in the case of the $A M F$. However, the difference is smaller than $2 \%$. All the solutions are able to reduce enormously the error in the estimation.

\section{Conclusions}

In this paper we presented the computation of the correction of the map when a robot closes a loop. The computation is carried out for three different methods based on the stochastic map approach. The first is the standard $E K F$ solution of $S L A M$ which updates a fully correlated covariance matrix, namely with a computational requirement $O\left(N^{2}\right)$. The second one is an approximation of the standard approach which updates only the correlations among landmarks close to each other. For this approximation we suggested a method to compute the correlations between the robot and all the

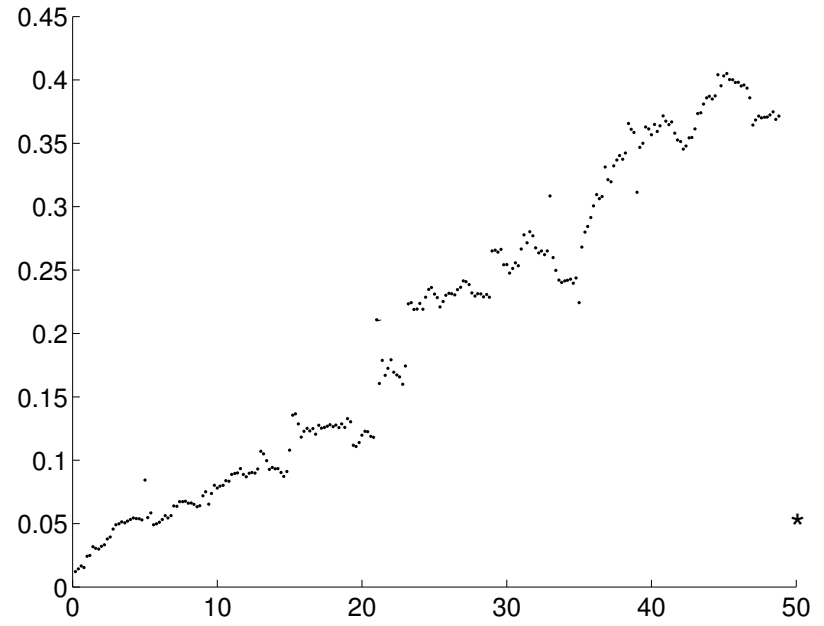

Fig. 2. The mean error on the landmarks in the simulated experiment, obtained by adopting the $A M F$. * indicates the error after the loop.

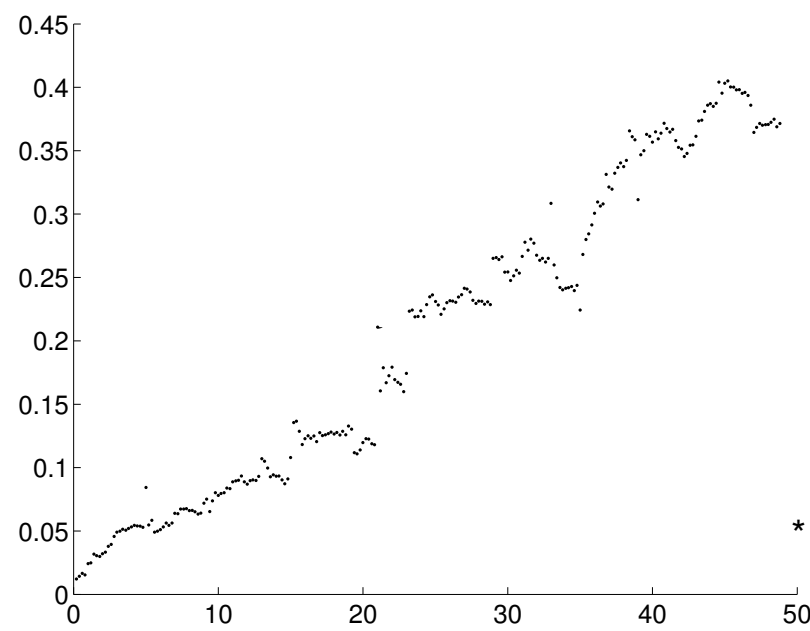

Fig. 3. The mean error on the landmarks in the simulated experiment, obtained by adopting the solution introduced in II-B. * indicates the error after the loop.

landmarks needed to propagate the correction along the loop. The computational requirement of this method is independent of the number of landmarks.

Finally, we compute for the same approximated solution the correlations by avoiding the linearization (which characterizes the Extended Kalman Filter and therefore the standard solution). This computation points out a very important result: the correlation between the robot and the landmarks are overestimated by the standard solution. In particular, the true correlation decreases exponentially with respect to the heading error of the robot.

The simulations confirm the results stated in the previous sections, namely that the approximated solution performs similarly to the standard one. 


\section{Acknowledgments}

This work has been supported by the two European projects RECSYS (Real-Time Embedded Control of Mobile Systems with Distributed Sensing) and BIBA (Bayesian Inspired Brain and Artefacts) and the Swiss project $F N$ (Scheizerischer Nationalfonds zur Forderung der Wissenschaftlichen Scientifique).

\section{REFERENCES}

[1] K.O. Arras, N. Tomatis, B.T. Jensen and R. Siegwart, "Multisensor onthe-fly localization: Precision and reliability for applications", Robotics and Autonomous Systems 34, pp. 131-143, 2001.

[2] Y. Bar-Shalom, T.E. Fortmann,, "Tracking and data association, mathematics in science and engineering", Vol 179, Academic Press, New York, 1988.

[3] J.A. Castellanos, J. Neira, J.D. Tardos, 2004, Limit to the Consistency of EKF-based SLAM , Intarnational Conference on Autonomous Vehicles (IAV), Lisbon Portugal, 2004

[4] K.S. Chong, L. Kleeman, "Accurate Odometry and Error Modelling for a Mobile Robot," International Conference on Robotics and Automation, vol. 4, pp. 2783-2788, 1997.

[5] J.L. Crowley, (1989). World Modeling and Position Estimation for a Mobile Robot Using Ultrasonic Ranging. IEEE International Conference on Robotics and Automation (ICRA), Scottsdale, AZ.

[6] Dissanayake, Newman, Clark, Durrant-Whyte and Csorba, 2001, A Solution to the Simultaneous Localization and Map Building (SLAM) problem, IEEE Trans. On Rob. And Aut. Vol 17, No.3, June 2001

[7] J.J. Leonard, H.F. Durrant-Whyte, "Directed Sonar Sensing for Mobile Robot Navigation," Kluwer Academic Publishers, Dordrecht, 1992.

[8] A. Martinelli, N. Tomatis, A. Tapus and R. Siegwart, "Simultaneous Localization and Odometry Calibration" International Conference on Inteligent Robot and Systems (IROSO3) Las Vegas, USA

[9] P.M. Newman, J.J. Leonard and R.J. Rikoski, "Towards Constant-Time SLAM on an Autonomous Underwater Vehicle Using Syntheic Aperture Sonar," International Symposyum of Robotics Research (ISRR03), October 2003, Siena, Italy.

[10] A. Papoulis, Probability, Random Variables, and Stochastic Process McGRAW-HILL INTERNATIONAL EDITIONS, 1991

[11] Smith, Self, et al. (1988) "Estimating uncertain spatial relationships in robotics" Uncertainty in Artificial Intelligence 2 Elsevier Science Pub: 435-461.

[12] S. Thrun, D. Koller, Z. Ghahramani, H.F. Durrant-Whyte and A.Y. Ng., "Simultaneous mapping and Localization with Sparse Extended Information Filters," Proceedings of the 5th Int. Workshop on Algorithmic Foundations of Robotics, Nice, France, 2002. 